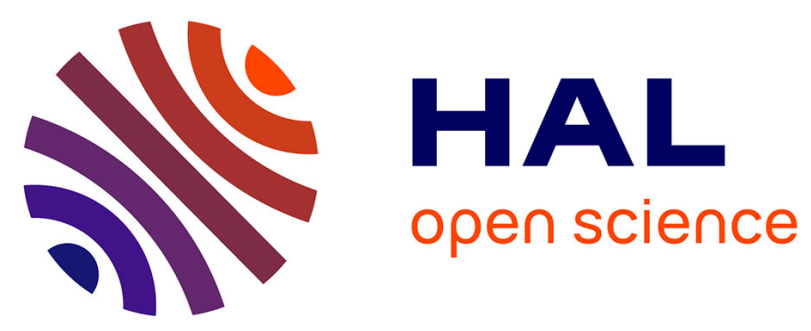

\title{
Analysis of PWA control of discrete-time linear dynamics in the presence of variable time-delay
}

Mohammed-Tahar Laraba, Sorin Olaru, Silviu-Iulian Niculescu

\section{To cite this version:}

Mohammed-Tahar Laraba, Sorin Olaru, Silviu-Iulian Niculescu. Analysis of PWA control of discretetime linear dynamics in the presence of variable time-delay. 55th IEEE Conference on Decision and Control (CDC 2016), Dec 2016, Las Vegas, United States. pp.567 - 572, 10.1109/CDC.2016.7798329 . hal-01429223

\section{HAL Id: hal-01429223}

\section{https://hal-centralesupelec.archives-ouvertes.fr/hal-01429223}

Submitted on 25 Mar 2017

HAL is a multi-disciplinary open access archive for the deposit and dissemination of scientific research documents, whether they are published or not. The documents may come from teaching and research institutions in France or abroad, or from public or private research centers.
L'archive ouverte pluridisciplinaire HAL, est destinée au dépôt et à la diffusion de documents scientifiques de niveau recherche, publiés ou non, émanant des établissements d'enseignement et de recherche français ou étrangers, des laboratoires publics ou privés. 


\title{
Analysis of PWA control of discrete-time linear dynamics in the presence of variable time-delay
}

\author{
Mohammed-Tahar Laraba, Sorin Olaru, and Silviu-Iulian Niculescu
}

\begin{abstract}
This paper focuses on the robustness problem for a specific class of dynamical systems, namely the piecewise affine (PWA) systems, defined over a bounded region of the state-space $\mathcal{X}$. We will be interested in PWA systems emerging from linear dynamical systems controlled via feedback channels in the presence of varying transmission delays by a PWA controller defined over a polyhedral partition of the state-space. We exploit the fact that the variable delays are inducing some particular model uncertainty. Our objective is to characterize the delay invariance margins: the collection of all possible values of the time-varying delays for which the positive invariance of $\mathcal{X}$ is guaranteed with respect to the closed-loop dynamics. These developments can be useful for the analysis of different design methodologies and in particular for predictive control approaches. The proposed delay margins describes the admissible transmission delays for an MPC implementation. From a different perspective, it further provides the fragility margins of an MPC implementation via the on-line optimization and subject to variable computational time.
\end{abstract}

\section{INTRODUCTION}

Time-delay dynamics form an important modeling class for networked control systems (NCSs) as well as for many other physical processes where propagation and transport phenomena, heredity and competition in population occur. The presence of communication networks in the closedloop induces varying transmission delays [1]. These delays are known to degrade the control performance and can induce instability as documented in the rich control literature dedicated to these subjects [2], [3].

Roughly speaking, Model Predictive Control (MPC) is a popular constrained control technique based on the resolution of an optimization problem over a receding horizon [4], [5]. It constructs at each sampling instant an optimal sequence with respect to a performance index. Unfortunately, using MPC in the presence of time-varying delays leads to complex optimization problems, which are difficult to handle. Linear MPC with constraints is known to result in PWA closed-loop dynamics [6], [7]. Recent work dealt with stability of PWA systems using Piecewise Quadratic Lyapunov functions [8]. The stability problem is usually formulated in terms of linear matrix inequalities (LMIs). Due to the conservatism of such approaches, alternative relaxations can be found in [9], [10].

In this paper, we will focus on the robustness analysis of discrete-time linear dynamics in closed-loop with a PWA control law in the presence of time-varying delays affecting the communication on the feedback channel or induced by

\footnotetext{
All authors are with the Laboratory of Signals and Systems of CentraleSupélec, Paris-Saclay University, 91190 Gif-SurYvette, France: mohammed.laraba, sorin.olaru, silviu.niculescullss.supelec.fr.
}

the control computation itself. The PWA feedback is generic but it can be obtained, for example, by using a simple explicit MPC design constructed upon the nominal delay-free model. A formal definition of delay margins based on positive invariance is one of the main objectives in this endeavor. The presence of variable input delay induces an uncertainty of exponential type in the closed-loop parameters. All possible delay variations can be covered by embedding the uncertainty within a polytopic model when the maximal delay is known [1], [11], [12]. However, the inverse problem of finding the maximal range of delay when a nonlinear control law structure is specified over a bounded region of the original state-space represents an open problem, to the best of the authors' knowledge, and will receive in the sequel a complete characterization.

The main contribution of the paper consists in proposing a constructive method to find the delay margins based on positive invariance of the nominal closed-loop dynamics in the piecewise affine formulation. The procedure describes, by means of set projections, all possible delay values for which the positive invariance (or alternatively $\mathcal{D}$-invariance) of the state trajectories is guaranteed. The paper makes use of some preliminary results in [13] and extends the robustness analysis to PWA systems with variable input delays.

The paper is structured as follows. The class of considered linear continuous time-invariant systems affected by variable delay is introduced in section II. In the same section, the exact discretization of the considered dynamics and the uncertain PWA system obtained in closed-loop are discussed. Section III is devoted to the construction of delay margins in order to ensure invariance in both the original and the extended state-space representations. Lastly, an illustrative example is shown in section IV and concluding remarks are drawn in section $\mathrm{V}$.

Notations: We denote by $\mathbb{R}, \mathbb{R}_{+}, \mathbb{N}, \mathbb{Z}$ and $\mathbb{Z}_{+}$the field of real numbers, the field of non-negative real numbers, the set of non-negative integers, the set of integer numbers and the set of strictly positive integer numbers, respectively. For every interval $\Pi$ of $\mathbb{R}$ we define $\mathbb{Z}_{\Pi}:=\mathbb{Z} \cap \Pi$. Given $m \in \mathbb{Z}_{+}$, by $\mathbb{1}_{m}$, we denote the vector of dimension $m$ with all the entries equal to 1 and, by $I_{m} \in \mathbb{R}^{m \times m}$, the $m \times m$ identity matrix. Conv denotes the convex hull operation.

Given two sets $\mathcal{X}, \mathcal{Y} \subset \mathbb{R}^{m}, \mathcal{X} \oplus \mathcal{Y}$ and $\mathcal{X} \times \mathcal{Y}$ denote the Minkowski sum and the Cartesian product of these two sets, respectively, defined as follows:

$$
\begin{gathered}
\mathcal{X} \oplus \mathcal{Y}:=\{z \mid \exists(x, y) \in(\mathcal{X}, \mathcal{Y}) \text { such that } z=x+y\} . \\
\mathcal{X} \times \mathcal{Y}:=\{(x, y) \mid x \in \mathcal{X} \text { and } y \in \mathcal{Y}\} .
\end{gathered}
$$


The unit simplex in $\mathbb{R}^{m}$ is defined as:

$$
S_{m}:=\left\{x \in \mathbb{R}_{+}^{m} \mid \mathbb{1}_{m}^{T} x=1\right\} .
$$

For a given set $\mathcal{X} \subset \mathcal{Y} \times \mathcal{Z}, \operatorname{int}(\mathcal{X})$ denotes the interior of $\mathcal{X}$, the projection of $\mathcal{X}$ onto $\mathcal{Y}$ is defined as:

$$
\operatorname{Proj}_{\mathcal{Y}} \mathcal{X}=\{y \in \mathcal{Y} \mid \exists z \in \mathcal{Z} \text { such that }(y, z) \in \mathcal{X}\} .
$$

The notions of state-space partition, PWA functions and positive invariance are the classical ones as defined for example in [13], [14]. Whenever an exponent is associated with a matrix, it will be interpreted as a matrix raised to a power or just an index depending on the context.

\section{DYNAMICAL MODEL OF A LINEAR PLANT WITH DIGITAL CONTROL IN THE PRESENCE OF INTERSAMPLE DELAY}

\section{A. Dynamical System}

Consider a nominal linear continuous time-invariant (LTI) system and a sequence of delays $\left(\tau_{k}\right)$ affecting the input as follows:

$$
\left\{\begin{array}{l}
\dot{x}(t)=A_{c} x(t)+B_{c} u(t) \\
u(t)=u_{k}, \forall t \in\left[t_{k}+\tau_{k}, t_{k+1}+\tau_{k+1}\right) .
\end{array}\right.
$$

where $A_{c} \in \mathbb{R}^{n \times n}, B_{c} \in \mathbb{R}^{n \times m}, x(t) \in \mathbb{R}^{n}$ the continuous system state. Moreover, assume that the system states are sampled periodically with the period $T_{s} \in \mathbb{R}_{>0}$ and we denote by $t_{k}=k T_{s}$ the $k^{t h}$ sampling instants. The control input $u(t) \in \mathbb{R}^{m}$ is known for $t \in\left[0, \tau_{0}\right)$, and the control action generated at time $t=t_{k}$ at the controller side is denoted by $u_{k} \in \mathbb{R}^{m}$. The possible delay induced by the network at sample instant $t_{k}$ is denoted by $\tau_{k} \in[\underline{\tau}, \bar{\tau}]$, with a lower bound $\underline{\tau} \in \mathbb{R}_{[0, \bar{\tau}]}$ and an upper bound $\bar{\tau} \in \mathbb{R}_{\left[\underline{\tau}, T_{s}\right]}$. For the sake of simplicity of the presentation, we discuss in the sequel only the case when the delay variation is intersample, i.e. smaller than or equal to the sampling period. We will recall next the modeling in discrete-time following the approach in the studies [1], [11], [12].

Consider the exact discretization of (1) by exploiting the fact that the control action is piecewise constant, i.e. $u(t)=$ $u_{k}, \forall t \in\left[t_{k}+\tau_{k}, t_{k+1}+\tau_{k+1}\right):$

$$
\begin{array}{r}
x_{k+1}=e^{A_{c} T_{s}} x_{k}+\int_{0}^{\tau_{k}} e^{A_{c}\left(T_{s}-\theta\right)} d \theta B_{c} u_{k-1}+ \\
\int_{\tau_{k}}^{T_{s}} e^{A_{c}\left(T_{s}-\theta\right)} d \theta B_{c} u_{k}
\end{array}
$$

and let $\epsilon_{k}=T_{s}-\tau_{k}$, and:

$$
\begin{gathered}
A=e^{A_{c} T_{s}}, B=\int_{0}^{T_{s}} e^{A_{c}\left(T_{s}-\theta\right)} d \theta B_{c} \\
\Delta\left(\epsilon_{k}\right)=\int_{T_{s}-\epsilon_{k}}^{T_{s}} e^{A_{c}\left(T_{s}-\theta\right)} d \theta B_{c}=\int_{-\epsilon_{k}}^{0} e^{-A_{c} \sigma} d \sigma B_{c} .
\end{gathered}
$$

Then, the discrete-time model which takes into account the effect of the continuous time-delay variation will become:

$$
x_{k+1}=A x_{k}+\Delta\left(\epsilon_{k}\right) u_{k}+\left(B-\Delta\left(\epsilon_{k}\right)\right) u_{k-1} .
$$

In the general case, the variable time delay implies a variable "limit" for the integration for $\epsilon_{k}$ in (4). One can see that there is no exact link between the samples available for the discrete model and the delay in continuous time, thus leading practically to some parameter-varying dynamical model. When discretizing, we can deal with the variable input delay as an appropriate uncertainty function. All possible delay variations can be covered by confining the induced model uncertainty within a polytopic description. Therefore, a polytopic (simplicial) over-approximation of the uncertainty coming from the variable delay can be constructed (See, e.g. [1], [12], [15]) to obtain finally a polytopic model. It is interesting to note that by setting $\epsilon_{k}=0$ and $\epsilon_{k}=T_{s}$, we obtain two extreme realizations of the discrete-time model (5).

\section{B. The PWA closed-loop dynamics}

The starting point for the present work will be the nominal dynamics corresponding to $\epsilon_{k}=T_{s}$ (no delay is induced by the network). An explicit PWA control law is designed with respect to this nominal dynamics.

$$
\begin{aligned}
& u_{\text {pwa }}: \mathcal{X} \longrightarrow \mathbb{R}^{m} \\
& u_{\text {pwa }}(x)=F_{i} x+g_{i}, \forall i \in \mathcal{I}_{N} \text { s.t } x \in \mathcal{X}_{i}
\end{aligned}
$$

The piecewise affine control law obtained will be in turn used in practice for the control of the linear parameter varying dynamics subject to variable delay (5). The closedloop dynamics resulting from applying:

$$
\left\{\begin{array}{l}
u_{p w a}^{k}=F_{i} x_{k}+g_{i}, \text { for } x_{k} \in \mathcal{X}_{i} \\
u_{p w a}^{k-1}=F_{j} x_{k-1}+g_{j}, \text { for } x_{k-1} \in \mathcal{X}_{j}
\end{array}\right.
$$

will be:

$$
\begin{gathered}
x_{k+1}=A x_{k}+\left(B-\Delta\left(\epsilon_{k}\right)\right)\left[F_{j} x_{k-1}+g_{j}\right]+ \\
\Delta\left(\epsilon_{k}\right)\left[F_{i} x_{k}+g_{i}\right] \\
=\left(A+\Delta\left(\epsilon_{k}\right) F_{i}\right) x_{k}+\left(B-\Delta\left(\epsilon_{k}\right)\right) F_{j} x_{k-1}+ \\
B g_{j}+\Delta(\epsilon)\left(g_{i}-g_{j}\right),
\end{gathered}
$$

$\forall(i, j) \in \mathcal{I}_{N}^{2}$ such that $x_{k} \in \mathcal{X}_{i}, x_{k-1} \in \mathcal{X}_{j}$.

It is clear that an extended state-space representation can be constructed for the delay-difference equation (8) by introducing some new augmented state vector, i.e. $\xi_{k}=$ $\left[x_{k}^{T} x_{k-1}^{T}\right]^{T} \in \mathbb{R}^{2 n}$. An equivalent extended state-space model will be obtained:

$$
\begin{array}{r}
\underbrace{\left[\begin{array}{c}
x_{k+1} \\
x_{k}
\end{array}\right]}_{\xi_{k+1}}=\left[\begin{array}{cc}
A+\Delta\left(\epsilon_{k}\right) F_{i} & \left(B-\Delta\left(\epsilon_{k}\right)\right) F_{j} \\
I_{n} & 0_{n \times n}
\end{array}\right] \underbrace{\left[\begin{array}{c}
x_{k} \\
x_{k-1}
\end{array}\right]}_{\xi_{k}}+ \\
{\left[\begin{array}{c}
B g_{j}+\Delta\left(\epsilon_{k}\right)\left(g_{i}-g_{j}\right) \\
0_{n \times 1}
\end{array}\right] ;} \\
\forall(i, j) \in \mathcal{I}_{N}^{2} \text { such that } x_{k} \in \mathcal{X}_{i} \text { and } x_{k-1} \in \mathcal{X}_{j} .
\end{array}
$$

The difference equations (8) and (9) depend on $\Delta\left(\epsilon_{k}\right)$, considered as a parameter varying matrix, lying in a nonconvex subset of $\mathbb{R}^{n \times m}$. One can write equations (8) and 
(9) in a more compact form as follows:

$$
\begin{array}{r}
x_{k+1}=\phi_{i}\left(\epsilon_{k}\right) x_{k}+\theta_{j}\left(\epsilon_{k}\right) x_{k-1}+\gamma_{i j}\left(\epsilon_{k}\right), \\
\forall(i, j) \in \mathcal{I}_{N}^{2} \text { s.t } x_{k} \in \mathcal{X}_{i} \text { and } x_{k-1} \in \mathcal{X}_{j} .
\end{array}
$$

where:

$$
\begin{aligned}
& \phi_{i}\left(\epsilon_{k}\right)=\left(A+\Delta\left(\epsilon_{k}\right) F_{i}\right) \\
& \theta_{j}\left(\epsilon_{k}\right)=\left(B-\Delta\left(\epsilon_{k}\right)\right) F_{j} \\
& \gamma_{i j}\left(\epsilon_{k}\right)=B g_{j}+\Delta\left(\epsilon_{k}\right)\left(g_{i}-g_{j}\right),
\end{aligned}
$$

or alternatively for (9) as:

$$
\left\{\begin{array}{l}
\xi_{k+1}=\Phi_{i j}\left(\epsilon_{k}\right) \xi_{k}+\Gamma_{i j}\left(\epsilon_{k}\right) \\
\forall(i, j) \in \mathcal{I}_{N}^{2} \text { such that } \xi_{k} \in \mathcal{X}_{i} \times \mathcal{X}_{j}
\end{array}\right.
$$

with:

$$
\begin{aligned}
\Phi_{i j}\left(\epsilon_{k}\right) & =\left[\begin{array}{cc}
A+\Delta\left(\epsilon_{k}\right) F_{i} & \left(B-\Delta\left(\epsilon_{k}\right)\right) F_{j} \\
I_{n} & 0_{n \times n}
\end{array}\right] \\
\Gamma_{i j}\left(\epsilon_{k}\right) & =\left[\begin{array}{c}
B g_{j}+\Delta\left(\epsilon_{k}\right)\left(g_{i}-g_{j}\right) \\
0_{n \times 1}
\end{array}\right]
\end{aligned}
$$

Moreover, one can define the following parameter-varying PWA mappings:

$$
\begin{aligned}
& \psi_{\text {pwa }}: \mathcal{X} \times \mathcal{X} \times \mathbb{R}_{\left[0, T_{s}\right]} \longrightarrow \mathbb{R}^{n} \\
& \left\{\begin{array}{l}
\psi_{\text {pwa }}(x, y, \epsilon)=\phi_{i}(\epsilon) x+\theta_{j}(\epsilon) y+\gamma_{i j}(\epsilon) \\
\forall(i, j) \in \mathcal{I}_{N}^{2} \text { such that } x \in \mathcal{X}_{i} \text { and } y \in \mathcal{X}_{j} .
\end{array}\right. \\
& \left\{\begin{array}{l}
\Psi_{\text {pwa }}:\{\mathcal{X} \times \mathcal{X}\} \times \mathbb{R}_{\left[0, T_{s}\right]} \longrightarrow \mathbb{R}^{2 n} \\
\Psi_{\text {pwa }}(\xi, \epsilon)=\Phi_{i j}(\epsilon) \xi+\Gamma_{i j}(\epsilon) \\
\forall(i, j) \in \mathcal{I}_{N}^{2} \text { such that } \xi \in \mathcal{X}_{i} \times \mathcal{X}_{j} .
\end{array}\right.
\end{aligned}
$$

It is worth to mention that the uncertain piecewise affine systems (10) and (12) are defined over the polyhedral partition of the compact sets $\mathcal{X}(\mathcal{X} \times \mathcal{X}$ respectively $), \mathcal{X}=\bigcup_{i \in \mathcal{I}_{N}} \mathcal{X}_{i}$ inherited from the explicit PWA control law design (6).

Now, the concept of delay margins with respect to dynamical systems, presented in the extended and the original statespace framework, is introduced in terms of sets as follows:

Definition 2.1: The delay margin with respect to the timevarying dynamical system (10), denoted $d_{m}$, is given by:

$$
d_{m}=\left\{\epsilon \in\left[0, T_{s}\right] \mid \forall x \in \mathcal{X}_{i}, y \in \mathcal{X}_{j}, \psi_{p w a}(x, y, \epsilon) \in \mathcal{X}\right\} .
$$

Definition 2.2: The delay margin with respect to the timevarying dynamical system (12), denoted $\mathcal{D}_{m}$, is given by:

$$
\mathcal{D}_{m}=\left\{\epsilon \in\left[0, T_{s}\right] \mid \forall \xi \in \mathcal{X} \times \mathcal{X}, \Psi_{\text {pwa }}(\xi, \epsilon) \in \mathcal{X} \times \mathcal{X}\right\} .
$$

To summarize, delay margin problem corresponds to the complete description of all possible delay values $\epsilon \in\left[0, T_{s}\right]$, denoted by $d_{m}$ (or by $\mathcal{D}_{m}$ ), for which the positive invariance of the set $\mathcal{X}$ (or alternatively $\mathcal{X} \times \mathcal{X}$ ) with respect to the time-varying dynamical systems (10), (alternatively (12)), is guaranteed, $\forall \epsilon \in d_{m}\left(\mathcal{D}_{m}\right)$, respectively.

\section{Construction OF THE DELAY MARGin BASED ON THE POSITIVE INVARIANCE}

Assume that both $\mathcal{X}$ and the elements $\mathcal{X}_{i}$ defining the partition in (6) are bounded polyhedral sets. Then, the polytopes of interest can be described as the intersection of finite number of half spaces. They can also be described as the convex hull of finite point set (vertices) in $\mathbb{R}^{n}$. Let the vertices of polytopes $\mathcal{X}$ and $\mathcal{X}_{i}$ be:

$$
\begin{gathered}
\mathcal{V}(\mathcal{X})=\left\{v_{1}, v_{2}, \cdots, v_{q}\right\} \\
\mathcal{V}\left(\mathcal{X}_{i}\right)=\left\{w_{i 1}, w_{i 2}, \cdots, w_{i q_{i}}\right\}, \forall i \in \mathcal{I}_{N}
\end{gathered}
$$

The vertex representation of these polytopes is expressed in the following:

$$
\begin{gathered}
\mathcal{X}=\operatorname{Conv}\left\{v_{1}, v_{2}, \cdots, v_{q}\right\} \\
\mathcal{X}_{i}=\operatorname{Conv}\left\{w_{i 1}, w_{i 2}, \cdots, w_{i q_{i}}\right\}, \forall i \in \mathcal{I}_{N}
\end{gathered}
$$

For each region $\mathcal{X}_{i}$ of the partition of $\mathcal{X}$, the set containing its vertices is:

$$
\mathcal{W}_{i}=\mathcal{V}\left(\mathcal{X}_{i}\right), \forall i \in \mathcal{I}_{N}
$$

Let $\mathcal{W}$ be the set of all vertices of $\mathcal{X}_{i}$ when $i \in \mathcal{I}_{N}$ :

$$
\mathcal{W}=\bigcup_{i \in \mathcal{I}_{N}} \mathcal{V}\left(\mathcal{X}_{i}\right)
$$

Using only the non redundant elements of $\mathcal{W}$, one can write:

$$
\mathcal{W}=\left\{w_{1}, w_{2}, \cdots, w_{p}\right\}, \operatorname{card}\{\mathcal{W}\}=p .
$$

The vertices of the polytope $\mathcal{X} \times \mathcal{X}$ are denoted by:

$$
\mathcal{V}(\mathcal{X} \times \mathcal{X})=\left\{\left(\begin{array}{c}
v_{i} \\
v_{j}
\end{array}\right) \in \mathbb{R}^{2 n}, \forall(i, j) \in \mathcal{I}_{q}^{2}\right\}
$$

For each region $\mathcal{X}_{i} \times \mathcal{X}_{j}$ of the partition of $\mathcal{X} \times \mathcal{X}$, its set of vertices:

$$
\begin{gathered}
\mathcal{W}_{i j}=\left[\mathcal{V}\left(\mathcal{X}_{i} \times \mathcal{X}_{j}\right)\right]=\mathcal{W}_{i} \times \mathcal{W}_{j} \\
\mathcal{W}_{i j}=\left\{w_{i 1}, w_{i 2}, \cdots, w_{i q_{i}}\right\} \times\left\{w_{j 1}, w_{j 2}, \cdots, w_{j q_{j}}\right\}
\end{gathered}
$$

Let $\mathcal{W}_{\mathcal{X} \times \mathcal{X}}$ be the set of all vertices of $\mathcal{X}_{i} \times \mathcal{X}_{j}$ when $(i, j) \in$ $\mathcal{I}_{N}^{2}$ :

$$
\mathcal{W}_{\mathcal{X} \times \mathcal{X}}=\bigcup_{(i, j) \in \mathcal{I}_{N}^{2}} \mathcal{V}\left(\mathcal{X}_{i} \times \mathcal{X}_{j}\right)
$$

It is worth to mention that there exists some close link between the elements of the two sets $\mathcal{W}$ and $\mathcal{W}_{\mathcal{X} \times \mathcal{X}}$. One can easily write:

$$
\mathcal{W}_{\mathcal{X} \times \mathcal{X}}=\left\{\left(\begin{array}{c}
w_{i} \\
w_{j}
\end{array}\right) \in \mathbb{R}^{2 n}, \forall(i, j) \in \mathcal{I}_{p}^{2}\right\} .
$$

Based on the above notations, we define the following matrices obtained by storing as columns the non redundant elements of the different sets of vertices using an arbitrary ordering:

$$
\begin{aligned}
& V=[\mathcal{V}(\mathcal{X})] \in \mathbb{R}^{n \times q}, \quad V_{\mathcal{X} \times \mathcal{X}}=[\mathcal{V}(\mathcal{X} \times \mathcal{X})] \in \mathbb{R}^{2 n \times q^{2}} \\
& V_{i}=\left[\mathcal{W}_{i}\right] \in \mathbb{R}^{n \times q_{i}}, \quad V_{i j}=\left[\mathcal{W}_{i j}\right] \in \mathbb{R}^{2 n \times\left(q_{i} \times q_{j}\right)} \\
& W=[\mathcal{W}] \in \mathbb{R}^{n \times p}, \quad W_{\mathcal{X} \times \mathcal{X}}=\left[\mathcal{W}_{\mathcal{X} \times \mathcal{X}}\right] \in \mathbb{R}^{2 n \times p^{2}}
\end{aligned}
$$

The image of the set $\mathcal{W}_{i}$ using the affine mapping (6) allows the construction of a matrix:

$$
U_{i}=\left[u_{p w a}\left(\mathcal{W}_{i}\right)\right] \in \mathbb{R}^{m \times q_{i}}
$$


The image of the set $\mathcal{W}$ via the affine mapping in (6) allows the construction of the matrix:

$$
U=\left[u_{p w a}(\mathcal{W})\right] \in \mathbb{R}^{m \times p} .
$$

Let $O_{p}^{k}$ be the $p \times p$ matrix whose all entries are equal to zero, except the $k^{t h}$ row, which is equal to $\mathbb{1}_{p}^{T}$.

A. Delay margins in the extended state-space representation

The uncertainty in (4) is represented by the matrix $\Delta\left(\epsilon_{k}\right)$ satisfying $\Delta\left(\epsilon_{k}\right) \in \boldsymbol{\Delta}, k \in \mathbb{N}$, with:

$$
\boldsymbol{\Delta}=\left\{\Delta\left(\epsilon_{k}\right) \mid \epsilon_{k} \in\left[0, T_{s}\right]\right\}
$$

To characterize the delay margins we aim to use a simplicial over-approximation of the matrices $\boldsymbol{\Delta} \in \mathbb{R}^{n \times m}$ in (30). Based on such an over-approximation of the matrix set $\Delta$, the system can be embedded within a polytopic model with $s+1$ extreme realizations:

$$
\boldsymbol{\Delta} \in \operatorname{Conv}\left\{\Delta_{0}, \Delta_{1}, \cdots, \Delta_{s}\right\},
$$

any element of $\boldsymbol{\Delta}$ can be written as convex combinations of generators (corresponding to extreme realizations), i.e.:

$$
\forall \epsilon_{k} \in \mathbb{R}_{\left[0, T_{s}\right]}, \exists \alpha \in S_{s+1} \text { such that } \Delta=\sum_{i=0}^{s} \alpha_{i} \Delta_{i} .
$$

More than that, with respect to the set $\mathcal{D}_{m} \subset \mathbb{R}_{\left[0, T_{s}\right]}$, we have:

$$
\forall \epsilon_{k} \in \mathcal{D}_{m}, \exists \alpha \in S_{s+1} \text { such that } \Delta=\sum_{i=0}^{s} \alpha_{i} \Delta_{i} .
$$

For a given $\xi_{k}$ such that $x_{k} \in \mathcal{X}_{i}$ and $x_{k-1} \in \mathcal{X}_{j}$, the feedback law is known i.e. $F_{i}, F_{j}, G_{i}$, and $G_{j}$ defining $\Phi_{i j}\left(\epsilon_{k}\right)$ and $\Gamma_{i j}\left(\epsilon_{k}\right)$ in (12) are known.

Proposition 3.1: For a given $(i, j) \in \mathcal{I}_{N}^{2}$, the matrix $\left[\Phi_{i j}\left(\epsilon_{k}\right) \Gamma_{i j}\left(\epsilon_{k}\right)\right]$ belongs to a polytopic set $\Omega$ :

$$
\Omega=\operatorname{Conv}\left\{\left[\Phi_{i j}^{1} \Gamma_{i j}^{1}\right], \cdots,\left[\Phi_{i j}^{s+1} \Gamma_{i j}^{s+1}\right]\right\}
$$

and there exists a vector $\alpha$ with non negative scalars $\left\{\alpha_{0}, \cdots, \alpha_{s}\right\}$ such that $\alpha \in S_{s+1}$ satisfying:

$$
\left[\Phi_{i j}\left(\epsilon_{k}\right) \Gamma_{i j}\left(\epsilon_{k}\right)\right]=\sum_{l=0}^{s} \alpha_{l}\left[\Phi_{i j}^{l} \Gamma_{i j}^{l}\right]
$$

where:

$$
\begin{gathered}
\Phi_{i j}^{l}=\left[\begin{array}{cc}
A+\Delta_{l} F_{i} & \left(B-\Delta_{l}\right) F_{j} \\
I_{n} & 0_{n \times n}
\end{array}\right], \\
\Gamma_{i j}^{l}=\left[\begin{array}{c}
B g_{j}+\Delta_{l}\left(g_{i}-g_{j}\right) \\
0_{n \times 1}
\end{array}\right] .
\end{gathered}
$$

The proof has been omitted due to space limitations.

Theorem 3.2: Consider the uncertain piecewise affine system (12) defined over the polyhedral partition of $\mathcal{X} \times \mathcal{X}$. The delay margin is obtained as:

$$
\mathcal{D}_{m}^{\alpha}=\left\{\operatorname{Proj}_{S_{s+1}} \mathcal{R}\right\} \cap \delta
$$

where $\mathcal{R}$ and $\delta$ are defined as:

$$
\delta=\left\{\alpha \in S_{s+1} \mid \forall \epsilon_{k} \in \mathbb{R}_{\left[0, T_{s}\right]} ; \Delta\left(\epsilon_{k}\right)=\sum_{i=0}^{s} \alpha_{i} \Delta_{i}\right\}
$$

$$
\begin{aligned}
& \mathcal{R}=\left\{(\alpha, \Gamma) \in \mathbb{R}_{+}^{s+1} \times \mathbb{R}_{+}^{q^{2} \times p^{2}} \mid \mathbb{1}^{T} \Gamma=\mathbb{1}^{T}\right. \\
& \left.E+\sum_{l=0}^{l=s} \alpha_{l}\left[\begin{array}{cc}
\Delta_{l} & 0_{n \times m} \\
0_{n \times m} & 0_{n \times m}
\end{array}\right] H=V_{\mathcal{X} \times \mathcal{X}} \Gamma\right\}
\end{aligned}
$$

$E \in \mathbb{R}^{2 n \times p^{2}}, H \in \mathbb{R}^{2 m \times p^{2}}$ are defined, next, in the proof.

Proof: The positive invariance of the set $\mathcal{X} \times \mathcal{X}$ with respect to the time-varying dynamical system (12) is represented by a set-wise relation:

$$
\begin{gathered}
\forall \epsilon_{k} \in \mathcal{D}_{m} \subset \mathbb{R}_{\left[0, T_{s}\right]}, \text { and } \forall \xi_{k} \in \mathcal{X}_{i} \times \mathcal{X}_{j},(i, j) \in \mathcal{I}_{N}^{2}: \\
\Phi_{i j}\left(\epsilon_{k}\right) \xi_{k}+\Gamma_{i j}\left(\epsilon_{k}\right) \in \mathcal{X} \times \mathcal{X}
\end{gathered}
$$

which is equivalent to:

$$
\forall \epsilon_{k} \in \mathcal{D}_{m} \subset \mathbb{R}_{\left[0, T_{s}\right]}, \text { and } \forall \xi_{k} \in \mathcal{X}_{i} \times \mathcal{X}_{j},(i, j) \in \mathcal{I}_{N}^{2},
$$$$
\exists \alpha \in S_{s+1} \text { such that } \sum_{l=0}^{s} \alpha_{l}\left[\Phi_{i j}^{l} \xi_{k}+\Gamma_{i j}^{l}\right] \in \mathcal{X} \times \mathcal{X} .
$$

By substituting (35) and (36) in equation (41) we obtain:

$$
\begin{gathered}
\sum_{l=0}^{s} \alpha_{l}\left[\left(\left[\begin{array}{cc}
A & B F_{j} \\
I_{n} & 0_{n \times n}
\end{array}\right]+\left[\begin{array}{cc}
\Delta_{l} & 0_{n \times m} \\
0_{n \times m} & 0_{n \times m}
\end{array}\right]\left[\begin{array}{cc}
F_{i} & -F_{j} \\
0_{m \times n} & 0_{m \times n}
\end{array}\right]\right)\right. \\
\left.\xi_{k}+\left[\begin{array}{c}
B g_{j} \\
0_{n \times 1}
\end{array}\right]+\left[\begin{array}{cc}
\Delta_{l} & 0_{n \times m} \\
0_{n \times m} & 0_{n \times m}
\end{array}\right]\left[\begin{array}{c}
g_{i}-g_{j} \\
0_{m \times 1}
\end{array}\right]\right] \in \mathcal{X} \times \mathcal{X} . \\
{\left[\begin{array}{cc}
A & B F_{j} \\
I_{n} & 0_{n \times n}
\end{array}\right] \xi_{k}+\left[\begin{array}{c}
B g_{j} \\
0_{n \times 1}
\end{array}\right]+\sum_{l=0}^{s} \alpha_{l}\left[\begin{array}{cc}
\Delta_{l} & 0_{n \times m} \\
0_{n \times m} & 0_{n \times m}
\end{array}\right]} \\
\left(\left[\begin{array}{cc}
F_{i} & -F_{j} \\
0_{m \times n} & 0_{m \times n}
\end{array}\right] \xi_{k}+\left[\begin{array}{c}
g_{i}-g_{j} \\
0_{m \times 1}
\end{array}\right]\right) \in \mathcal{X} \times \mathcal{X} .
\end{gathered}
$$

By expressing the extended state vector $\xi_{k} \in \mathcal{X}_{i} \times \mathcal{X}_{j}$ as a convex combinations of the vertices of $\mathcal{X}_{i} \times \mathcal{X}_{j}$ which is known to be polyehdral set, we obtain:

$$
\xi_{k}=\sum_{z=1}^{q_{i} \times q_{j}} \beta_{z} w_{i j}^{z} \text { for } \beta \in S_{q_{i} \times q_{j}}
$$

It follows that equation (43) is equivalent with:

$$
\begin{array}{r}
{\left[\begin{array}{cc}
A & B F_{j} \\
I_{n} & 0_{n \times n}
\end{array}\right] \sum_{z=1}^{q_{i} \times q_{j}} \beta_{z} w_{i j}^{z}+\left[\begin{array}{c}
B g_{j} \\
0_{n \times 1}
\end{array}\right]+\sum_{l=0}^{s} \alpha_{l}\left[\begin{array}{cc}
\Delta_{l} & 0_{n \times m} \\
0_{n \times m} & 0_{n \times m}
\end{array}\right]} \\
\quad\left(\left[\begin{array}{cc}
F_{i} & -F_{j} \\
0_{m \times n} & 0_{m \times n}
\end{array}\right] \sum_{z=1}^{q_{i} \times q_{j}} \beta_{z} w_{i j}^{z}+\left[\begin{array}{c}
g_{i}-g_{j} \\
0_{m \times 1}
\end{array}\right]\right) \in \mathcal{X} \times \mathcal{X} .
\end{array}
$$

For a given vertex in $w_{i j}^{z}$, i.e. $z \in \mathbb{Z}_{\left[1, q_{i} \times q_{j}\right]},(i, j) \in \mathcal{I}_{N}^{2}$ we have:

$$
\begin{array}{r}
{\left[\begin{array}{cc}
A & B F_{j} \\
I_{n} & 0_{n \times n}
\end{array}\right] w_{i j}^{z}+\left[\begin{array}{c}
B g_{j} \\
0_{n \times 1}
\end{array}\right]+\sum_{l=0}^{s} \alpha_{l}\left[\begin{array}{cc}
\Delta_{l} & 0_{n \times m} \\
0_{n \times m} & 0_{n \times m}
\end{array}\right]} \\
\left(\left[\begin{array}{cc}
F_{i} & -F_{j} \\
0_{m \times n} & 0_{m \times n}
\end{array}\right] w_{i j}^{z}+\left[\begin{array}{c}
g_{i}-g_{j} \\
0_{m \times 1}
\end{array}\right]\right) \in \mathcal{X} \times \mathcal{X} .
\end{array}
$$


We describe the inclusion (46) explicitly since it is equivalent with the existence of a vector $y_{i j}^{z} \in \mathcal{X} \times \mathcal{X}$ such that:

$$
\begin{array}{r}
{\left[\begin{array}{cc}
A & B F_{j} \\
I_{n} & 0_{n \times n}
\end{array}\right] w_{i j}^{z}+\left[\begin{array}{c}
B g_{j} \\
0_{n \times 1}
\end{array}\right]+\sum_{l=0}^{s} \alpha_{l}\left[\begin{array}{cc}
\Delta_{l} & 0_{n \times m} \\
0_{n \times m} & 0_{n \times m}
\end{array}\right]} \\
\left(\left[\begin{array}{cc}
F_{i} & -F_{j} \\
0_{m \times n} & 0_{m \times n}
\end{array}\right] w_{i j}^{z}+\left[\begin{array}{c}
g_{i}-g_{j} \\
0_{m \times 1}
\end{array}\right]\right)=y_{i j}^{z} .
\end{array}
$$

where the vector $y_{i j}^{z}$ can be expressed as:

$$
y_{i j}^{z}=V_{\mathcal{X} \times \mathcal{X}} \gamma_{i j}^{z} \text { such that } \gamma_{i j}^{z} \in S_{q^{2}}
$$

replacing equations (48) in (47), $\forall(i, j) \in \mathcal{I}_{N}^{2}$ and $z \in$ $\mathbb{Z}_{\left[1, q_{i} \times q_{j}\right]}$, we obtain:

$$
\begin{gathered}
{\left[\begin{array}{cc}
A & B F_{j} \\
I_{n} & 0_{n \times n}
\end{array}\right] w_{i j}^{z}+\left[\begin{array}{c}
B g_{j} \\
0_{n \times 1}
\end{array}\right]+\sum_{l=0}^{s} \alpha_{l}\left[\begin{array}{cc}
\Delta_{l} & 0_{n \times m} \\
0_{n \times m} & 0_{n \times m}
\end{array}\right]} \\
\left(\left[\begin{array}{cc}
F_{i} & -F_{j} \\
0_{m \times n} & 0_{m \times n}
\end{array}\right] w_{i j}^{z}+\left[\begin{array}{c}
g_{i}-g_{j} \\
0_{m \times 1}
\end{array}\right]\right)=V_{\mathcal{X} \times \mathcal{X} \gamma_{i j}^{z} .}
\end{gathered}
$$

or in other words, equation (49) holds for all non redundant vertices of $\mathcal{X}_{i} \times \mathcal{X}_{j}, \forall(i, j) \in \mathcal{I}_{N}^{2}$, which means that it holds for all the columns of the matrix $W_{\mathcal{X} \times \mathcal{X}}$ defined in (27). Exploiting the piecewise affine mapping (6) of the elements of $\mathcal{W}_{\mathcal{X} \times \mathcal{X}}$, a matrix formulation of the elements

$$
\begin{gathered}
{\left[\begin{array}{cc}
A & B F_{j} \\
I_{n} & 0_{n \times n}
\end{array}\right] w_{i j}^{z}+\left[\begin{array}{c}
B g_{j} \\
0_{n \times 1}
\end{array}\right],} \\
{\left[\begin{array}{cc}
F_{i} & -F_{j} \\
0_{m \times n} & 0_{m \times n}
\end{array}\right] w_{i j}^{z}+\left[\begin{array}{c}
g_{i}-g_{j} \\
0_{m \times 1}
\end{array}\right]}
\end{gathered}
$$

in (49) is deduced for $w_{i j}^{z} \in \mathcal{W}_{\mathcal{X} \times \mathcal{X}}$ :

$$
\begin{aligned}
& \left\{\begin{array}{l}
{\left[\begin{array}{cc}
A & B F_{j} \\
I_{n} & 0_{n \times n}
\end{array}\right] w_{i j}^{z}+\left[\begin{array}{c}
B g_{j} \\
0_{n \times 1}
\end{array}\right]=} \\
w_{i j}^{z} \in \mathcal{W}_{\mathcal{X} \times \mathcal{X}}
\end{array}\right. \\
& {\left[\begin{array}{cc}
A & 0_{n \times n} \\
I_{n} & 0_{n \times n}
\end{array}\right] W_{\mathcal{X} \times \mathcal{X}}+\left[\begin{array}{c}
B U \\
0_{n \times p}
\end{array}\right]\left[\begin{array}{lll}
I_{p} & \cdots & I_{p}
\end{array}\right]}
\end{aligned}
$$

Same procedure can be applied for (51):

$$
\begin{aligned}
& \left\{\begin{array}{cc}
{\left[\begin{array}{cc}
F_{i} & -F_{j} \\
0_{m \times n} & 0_{m \times n}
\end{array}\right] w_{i j}^{z}+\left[\begin{array}{c}
g_{i}-g_{j} \\
0_{m \times 1}
\end{array}\right]=} \\
w_{i j}^{z} \in \mathcal{W}_{\mathcal{X} \times \mathcal{X}}
\end{array}=\right. \\
& {\left[\begin{array}{ccc}
U O_{p}^{1}-U & U O_{p}^{2}-U & \cdots U O_{p}^{p}-U \\
0_{m \times p} & 0_{m \times p} & \cdots 0_{m \times p}
\end{array}\right]}
\end{aligned}
$$

and

$$
\left\{\begin{array}{l}
V_{\mathcal{X} \times \mathcal{X}} \gamma_{i j}^{z} \\
w_{i j}^{z} \in \mathcal{W}_{\mathcal{X} \times \mathcal{X}}
\end{array}=V_{\mathcal{X} \times \mathcal{X}} \Gamma\right.
$$

with the restriction that each column of $\Gamma$ is restricted to $S_{q^{2}}$. This restriction can be expressed as linear constraints on the columns of $\Gamma$ :

$$
\mathbb{1}^{T} \Gamma=\mathbb{1}^{T}, \Gamma \in \mathbb{R}_{+}^{q^{2} \times p^{2}}
$$

Finally, equation (49) leads to the matrix formulation:

$$
\underbrace{\left[\begin{array}{cc}
A & 0_{n \times n} \\
I_{n} & 0_{n \times n}
\end{array}\right] W_{\mathcal{X} \times \mathcal{X}}+\left[\begin{array}{c}
B U \\
0_{n \times p}
\end{array}\right]\left[\begin{array}{lll}
I_{p} & \cdots & I_{p}
\end{array}\right]}_{E}+
$$

$$
\begin{gathered}
\sum_{l=0}^{l=s} \alpha_{l}\left[\begin{array}{cc}
\Delta_{l} & 0_{n \times m} \\
0_{n \times m} & 0_{n \times m}
\end{array}\right] \underbrace{\left[\begin{array}{ccc}
U O_{p}^{1}-U & \cdots & U O_{p}^{p}-U \\
0_{m \times p} & \cdots & 0_{m \times p}
\end{array}\right]}_{H} \\
=V_{\mathcal{X} \times \mathcal{X} \Gamma} \\
E+\sum_{l=0}^{l=s} \alpha_{l}\left[\begin{array}{cc}
\Delta_{l} & 0_{n \times m} \\
0_{n \times m} & 0_{n \times m}
\end{array}\right] H=V_{\mathcal{X} \times \mathcal{X} \Gamma .}
\end{gathered}
$$

Since the exponential uncertainty corresponds to the values of $\alpha$ in (38), $\left(\Delta\left(\epsilon_{k}\right)\right.$ does not take all values in the embedding), the delay margin is obtained as (37) in terms of $\alpha$. the proof is complete noticing that the two sets $\mathcal{D}_{m}^{\alpha}$ and $\mathcal{D}_{m}$ are isomorphic.

\section{B. Delay margins in the original state-space representation}

Theorem 3.3: Consider the uncertain piecewise affine systems (10) defined over the polyhedral partition of $\mathcal{X}$. The delay margin $d_{m}$ is obtained as follows:

$$
d_{m}^{\alpha}=\left\{\operatorname{Proj}_{S_{s+1}} \mathcal{T}\right\} \cap \delta
$$

where $\delta$ is defined in (38) and $\mathcal{T}$ is defined as:

$$
\begin{array}{r}
\mathcal{T}=\left\{(\alpha, L) \in \mathbb{R}_{+}^{s+1} \times \mathbb{R}_{+}^{q \times p^{2}} \mid \mathbb{1}^{T} L=\mathbb{1}^{T},\right. \\
\left.E^{\prime}+\sum_{l=0}^{l=s} \alpha_{l} \Delta_{l} H^{\prime}=V L\right\}
\end{array}
$$

and

$$
\begin{gathered}
E^{\prime}=\left[\begin{array}{ll}
A W O_{p}^{1}+B U & \cdots A W O_{p}^{p}+B U
\end{array}\right], \\
H^{\prime}=\left[\begin{array}{ll}
U O_{p}^{1}-U & \cdots U O_{p}^{p}-U
\end{array}\right] .
\end{gathered}
$$

The proof has been omitted here due to space limitations.

\section{ILLUSTRATIVE EXAMPLE}

Consider the following unstable dynamical system:

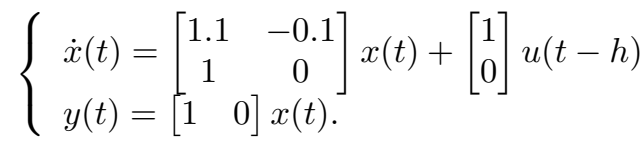

with $h \in[0,0.1]$. A discrete model is obtained, using (2)-(4) with a sampling period $T_{s}=0.1$, and the uncertainty $0<$ $\epsilon_{k} \leq 0.1$. The uncertainty matrix $\Delta\left(\epsilon_{k}\right)$ has been embedded within a polytopic model with the following 3 vertices:

$$
\Delta \in \operatorname{Conv}\left\{\left[\begin{array}{l}
0 \\
0
\end{array}\right],\left[\begin{array}{c}
0.0999 \\
-0.0006
\end{array}\right],\left[\begin{array}{l}
0.1057 \\
0.0057
\end{array}\right]\right\}
$$

Then, an explicit MPC has been designed for the nominal model (delay-free, $h=0$ ), with a prediction horizon $N=7$, in the presence of input and output constraints:

$$
\begin{aligned}
& -5 \leq u_{k} \leq 5 \\
& -5 \leq y_{k} \leq 5
\end{aligned}
$$

The partition of the obtained PWA control law as well as the resulting over-approximation of the uncertainty are shown in Figure 1.

The delay margin $d_{m}^{\alpha}$ has been computed using (57). Its projection on the plane $\left(\alpha_{0}, \alpha_{1}\right)$ is shown in Figure 2. The red set and the curved black line represent the sets $\mathcal{T}$ and $\delta$ 

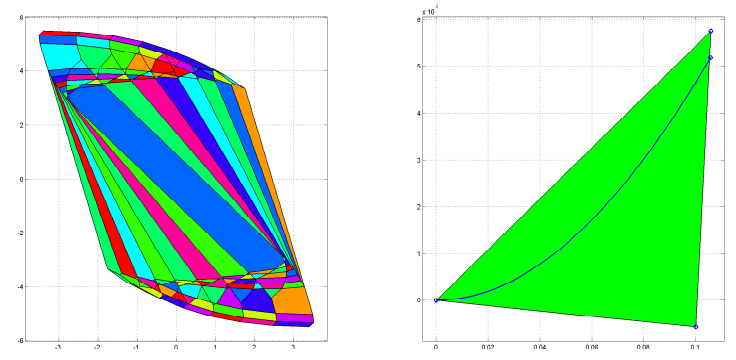

Fig. 1. Partition of the PWA control law (left), illustration of the embedding of $\Delta\left(\epsilon_{k}\right)$ by a simplex (right)

defined in (58) and (38), respectively, both projected on $S_{2}$. Finally the delay margin is obtained $d_{m}=[0.0972 ; 0.1]$, which corresponds to a delay variation $\tau_{k} \in[0 ; 0.0028]$.

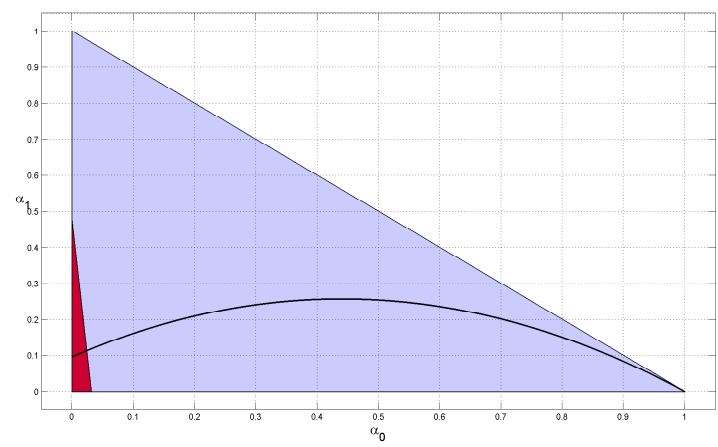

Fig. 2. Delay margin $d_{m}^{\alpha}$ projected on the plane $\left(\alpha_{0}, \alpha_{1}\right)$

Note that some close link between the delay margins and the number of regions in the partition of the PWA control law exists. The number of regions being directly related to the choice of the prediction horizon, one can notice that the delay margin is larger when the prediction horizon is small. Delay margins for different PWA control laws obtained using different prediction horizons are shown in Table I.

TABLE I

DELAY MARGINS AND THE PREDICTION HORIZON

\begin{tabular}{|c|c|c|c|c|c|}
\hline$N$ & 2 & 3 & 4 & 5 & 6 \\
\hline$\tau_{k} \times 10^{3}$ & {$[0 ; 5.6]$} & {$[0 ; 4.9]$} & {$[0 ; 4.2]$} & {$[0 ; 3.7]$} & {$[0 ; 3.2]$} \\
\hline
\end{tabular}

\section{CONCLUSION}

In discrete-time modeling framework, the analysis of linear systems affected by uncertain time-varying input delays passes through the characterization of uncertain delay difference equations depending on a parameter varying matrix. The uncertainty appears in the parametrization of an exponential matrix. Many different techniques exist in the literature aiming at constructing an embedding for the uncertainty, and leading to classical polytopic models.
In the present work, we addressed an inverse problem, offering a measure of the delay margin of positive invariance for a closed-loop PWA system in the original (related to $\mathcal{D}$ invariance) and the extended state-space representations. The result presented in this paper gives a way to tackle the delay margin problem of a nominal PWA control law which can be seen as a relevant issue from the robustness analysis point of view in both feedback communication channels and variable computation-time for real-time optimization-based control.

\section{ACKNOWLEDGMENTS}

The research leading to these results has benefited from the financial support of the European Union's $7^{\text {th }}$ Framework Programme under EC-GA No. 607957 TEMPO - Training in Embedded Predictive Control and Optimization.

\section{REFERENCES}

[1] W. Heemels, N. Van de Wouw, R. Gielen, M. Donkers, L. Hetel, S. Olaru, M. Lazar, J. Daafouz, and S. Niculescu, "Comparison of overapproximation methods for stability analysis of networked control systems," in Proceedings of the $13^{\text {th }}$ ACM International Conference on Hybrid systems: computation and control, 2010, pp. 181-190.

[2] W. Michiels and S.-I. Niculescu, Stability and Stabilization of TimeDelay Systems (Advances in Design \& Control). Philadelphia, PA, USA: Society for Industrial and Applied Mathematics, 2007.

[3] K. Gu, V. L. Kharitonov, and J. Chen, Stability of time-delay systems. Birkhäuser, Boston, USA: Springer Science + Business Media, LLC, 2003

[4] J. Maciejowski, Predictive Control with Constraints. England.: Prentice Hall, 2002.

[5] T. A. Johansen and A. Grancharova, Explicit Nonlinear Model Predictive Control. Springer-Verlag Berlin Heidelberg, 2012.

[6] A. Bemporad, M. Morari, V. Dua, and E. N. Pistikopoulos, "The explicit linear quadratic regulator for constrained systems," Automatica, vol. 38, no. 1, pp. 3-20, 2002.

[7] T. A. Johansen and A. Grancharova, "Approximate explicit constrained linear model predictive control via orthogonal search tree," IEEE Transactions on Automatic Control, vol. 48, no. 5, pp. 810-815, 2003.

[8] M. Johansson and A. Rantzer, "Computation of piecewise quadratic lyapunov functions for hybrid systems," IEEE Transactions on Automatic Control, vol. 43, no. 4, pp. 555-559, 1998.

[9] M. Hovd and S. Olaru, "Relaxing PWQ lyapunov stability criteria for PWA systems," Automatica, vol. 49, no. 2, pp. 667-670, 2013.

[10] R. Iervolino, F. Vasca, and L. Iannelli, "Cone-copositive piecewise quadratic lyapunov functions for conewise linear systems," IEEE Transactions on Automatic Control, vol. 60, no. 11, pp. 3077-3082, 2015.

[11] M. B. G. Cloosterman, N. van de Wouw, W. M. P. H. Heemels, and H. Nijmeijer, "Robust stability of networked control systems with time-varying network-induced delays," in $45^{\text {th }}$ IEEE Conference on Decision and Control, 2006, pp. 4980-4985.

[12] R. Gielen, S. Olaru, M. Lazar, W. Heemels, N. Van de Wouw, and S. Niculescu, "On polytopic inclusions as a modeling framework for systems with time-varying delays," Automatica, vol. 46, no. 3, pp. 615-619, 2010

[13] N. A. Nguyen, S. Olaru, P. Rodriguez-Ayerbe, G. Bitsoris, and M. Hovd, "Explicit robustness and fragility margins for linear discrete systems with piecewise affine control law," Automatica, vol. 68, pp. 334-343, 2016.

[14] F. Blanchini, "Set invariance in control," Automatica, vol. 35, pp $1747-1767,1999$.

[15] W. Lombardi, S. Olaru, S.-I. Niculescu, and L. Hetel, "A predictive control scheme for systems with variable time-delay," International Journal of Control, vol. 85, no. 7, pp. 915-932, 2012. 